Check for updates

Cite this: RSC Adv., 2017, 7, 51838

Received 11th October 2017

Accepted 2nd November 2017

DOI: $10.1039 / c 7 r a 11188 f$

rsc.li/rsc-advances

\section{Sheetlike gold nanostructures/graphene oxide composites via a one-pot green fabrication protocol and their interesting two-stage catalytic behaviors $\uparrow$}

\author{
Guangwei Geng, ${ }^{\text {ab }}$ Penglei Chen, (D) *abc Bo Guan, ${ }^{\mathrm{b}}$ Yu Liu, ${ }^{\text {ab }}$ Changchun Yang, ${ }^{\mathrm{a}}$ \\ Nannan Wang ${ }^{b}$ and Minghua Liu (D) bc
}

\begin{abstract}
Au nanoarchitectures shaped with a sheetlike morphology have drawn great attention owing to their potential applications in diverse areas of paramount importance. While great achievements have been made in the fabrication of Au nanoplates, most of the currently existing synthesis protocols generally suffer from either the requirement of rigorous reaction conditions, the involvement of specific apparatus, the use of harmful chemicals, etc. The initiation of an easy, environmentally benign yet low cost method to avoid these issues is strongly desired. In this contribution, we find that Au nanoplates could be fabricated under ambient conditions simply by mixing an aqueous solution of potassium tetrachloroaurate(III), an aqueous dispersion of graphene oxide (GO), and ethanol. Interestingly, we show that the as-manufactured $\mathrm{Au}$ nanoplate/GO composite displays fascinating two-stage catalytic performance towards $\mathrm{NaBH}_{4}$-induced reduction of 4-nitrophenol (4-NP), in which the catalytic reactivity of the latter stage exhibits a substantial enhancement by a factor of ca. 9 times compared to that of the former stage. Our investigation suggests that such fascinating catalytic behaviors could be ascribed to the $\mathrm{NaBH}_{4}$-induced restoration of the $\pi$-conjugated aromatic network of $\mathrm{GO}$ during the first stage, which promotes electron transfer from GO to Au nanoplates and facilitates the anchoring of 4-NPs onto the composites, resulting in the dramatically boosted catalytic performance of the second stage. This work might not only introduce a facile, green yet low-cost protocol for the synthesis of Au nanoplates but also provide deep insights into the catalytic performances of GO-based hybrid catalysts in terms of an interesting two-stage catalytic behavior.
\end{abstract}

\section{Introduction}

In recent years, anisotropic Au nanostructures of a well-defined yet unique morphology have been the focus of much attention because of their fascinating shape-sensitive physicochemical attributes and functions, which make them promising candidates in various areas of paramount importance. ${ }^{\mathbf{1 - 4}}$ Among the diverse anisotropic Au nanostructures, those characterized with a two-dimensional platelike architecture have been attracting increasing interest and become a hot topic of this field..$^{5-21}$ This

${ }^{a}$ College of Chemistry and Molecular Engineering, Zhengzhou University, 100 Science Road, Zhengzhou 450001, Henan, China.E-mail: cpl@zzu.edu.cn

${ }^{b}$ Beijing National Laboratory for Molecular Science, CAS Key Lab of Colloid, Interface and Chemical Thermodynamics, CAS Research/Education Center for Excellence in Molecular Sciences, Institute of Chemistry, Chinese Academy of Sciences, Beijing 100190, China. E-mail: chenpl@iccas.ac.cn

'University of Chinese Academy of Sciences, Beijing 100049, China

$\dagger$ Electronic supplementary information (ESI) available: The SEM image of our nanostructures without GO nanosheets, and real-time UV-vis spectra of the reduction of 4-NP. See DOI: 10.1039/c7ra11188f is owing to their intrinsic features, such as high aspect ratio, high anisotropy, the existence of fertile sharp corners and edges, etc. ${ }^{22-26}$ which endow Au nanoplates with unique optical, electrical, and mechanical properties. ${ }^{5-26}$ These inherent attributes provide Au nanoplates with great opportunities in a wide variety of fields, including but not confined to catalysis, energy conversion, optoelectronics, information storage, sensors, cancer hyperthermia, imaging, surface-enhanced Raman scattering, etc. ${ }^{5-28}$ So far, numerous efforts have been successfully devoted to the synthesis of $\mathrm{Au}$ nanoplates, wherein various photochemical, electrochemical, biochemical, thermal, and polyol methods, and seed-mediated growth protocols etc., are frequently used. ${ }^{53}$ Nevertheless, either owing to the involvement of toxic chemicals, the necessity of rigorous reaction conditions or a careful and accurate control of the experimental parameters, the requirement of templates or capping reagents, or the use of specific apparatus, etc., most of the currently existing synthesis strategies are either laborious, tedious, complex, painstaking, or high cost. It still remains a formidable challenge to initiate a facile, low cost yet environmentally 
benign method for the manufacturing of $\mathrm{Au}$ nanoplates, wherein the above-addressed limitations could be evaded to some extent.

On another front, graphene oxide (GO), which is geometrically an atomic thick nanosheet of covalently arranged honeycomb lattice of carbon atoms and whose basal plane and edge are decorated with various oxygen-containing functional groups, have recently received considerable attention as one of the most frequently addressed graphene cousins. ${ }^{34,35}$ The coexistence of oxygen functionalities and locally distributed polyaromatic graphene fragments on its framework endow it with versatile opportunities for the construction of GO-based hybrid materials, wherein the properties/functions of the individual components could be efficiently tuned and/or enhanced..$^{34,35}$ In addition to the great achievements with respect to GO-based advanced functional materials, ${ }^{34,35}$ it has currently been recognized that GO could also work as an ideal two-dimensional template to direct the synthesis of platelike nanostructures of noble metals. So far, numerous nanoplates of Ag, Pt, and Au have been successfully formulated via the twodimensional template effect of GO. ${ }^{36-43}$ Nevertheless, in these cases, the fabrication has to be fulfilled with the assistance of appropriate alkylamine, ${ }^{36,37}$ polyethylenimine,$^{38}$ tannic acid, ${ }^{39}$ a molten salt medium, ${ }^{\mathbf{4 0}}$ a heating or hydrothermal treatment, ${ }^{\mathbf{4 1 4 2}}$ or an electrochemical process. ${ }^{\mathbf{4 3}}$ It thus is strongly desired to launch a facile yet green protocol to produce $\mathrm{Au}$ nanoplates/GO composites under ambient conditions without the use of non-environmentally friendly chemicals or specific apparatus.

Inspired by the above-mentioned brief backgrounds, we herein report that Au nanoplates/GO composites could be easily fabricated via a one-pot GO-templated method under ambient conditions. We show that simply by means of mixing an aqueous solution of potassium tetrachloroaurate(III), an aqueous dispersion of GO, and ethanol, which serve as $\mathrm{Au}$ source, two-dimensional template, and reducing agent, respectively, Au nanoplates could be manufactured at room temperature. It is disclosed that the as-fabricated Au nanoplates are single-crystalline with the (111) facets as their basal plane. Interestingly, our Au nanoplates/GO composites exhibit a twostage catalytic behavior towards the catalytic reduction of 4nitrophenol (4-NP), in which the catalytic reactivity of the latter stage increases substantially by a factor of $c a$. 9 times compared to that of the former stage. It has been verified that the enhanced catalytic performances of the latter stage could be attributed to the $\mathrm{NaBH}_{4}$-induced reduction of GO during the former stage, wherein the synergistic effects of the promoted electron transfer from GO to Au nanoplates and the facilitated anchoring of 4-NP molecules onto the composites, play an important role. Our work might provide a facile, low-cost, and green method for the fabrication of $\mathrm{Au}$ nanoplates/GO composites. Meanwhile, in terms of an interesting two-stage catalytic phenomenon, our investigation likely provides deep insights into the catalytic performances of GO-hybridized catalysts, which is a topic of paramount significant and general interest.

\section{Experimental section}

\subsection{Chemicals and reagents}

Potassium tetrachloroaurate(III) $\left(\mathrm{KAuCl}_{4}\right.$, Alfa Aesar, 99\%), graphite powder (Alfa Aesar, 325 mesh, 99.99\%), 4-nitrophenol (4-NP, Beijing Chemical Reagents Plant, A. R.), sodium borohydride $\left(\mathrm{NaBH}_{4}\right.$, Sigma-Aldrich, $\geq 99 \%$ ), and ethanol (Beijing Chemical Works, $\geq 99.7 \%$ ) were used as received without further treatment.

\subsection{Preparation of graphene oxide (GO) nanosheets}

GO nanosheets were synthesized via a chemical exfoliation of graphite powder by means of a modified Hummers' method. ${ }^{\mathbf{4 4 , 4 5}}$ The detailed fabrication process was carried out according to the procedures described previously. ${ }^{46}$

\subsection{Synthesis of Au nanoplates/GO composites}

In a typical synthesis, $20 \mathrm{~mL}$ ethanol and $10 \mathrm{~mL}$ aqueous dispersion of $\mathrm{GO}$ nanosheets $\left(1 \mathrm{mg} \mathrm{mL}^{-1}\right)$ were mixed with $65 \mathrm{~mL}$ Milli-Q water. Then, a $5 \mathrm{~mL}$ aqueous solution of $\mathrm{KAuCl}_{4}$ $\left(1 \times 10^{-3} \mathrm{~mol} \mathrm{~L}^{-1}\right)$ was added dropwise into the above dispersion. The as-obtained systems were aged under ambient conditions for ten days, after which the products were collected and washed adequately with Milli-Q water by means of repeating high-speed centrifugation $(12000 \mathrm{rpm}, 15$ minutes, the yield of the composites was estimated to be $c a .1 .2 \mathrm{mg}$ ). Subsequently, the as-prepared samples were used for various characterizations or for catalytic performances. Controlled experiments without the use of GO nanosheets were also conducted for comparison, wherein nearly similar operations were carried out except that identical volume of Milli-Q water $(10 \mathrm{~mL})$ instead of GO dispersions was added into the preparation system. To monitor the evolution process of the synthesis, the reaction was terminated by means of filtration at a desired aging stage. After being adequately washed with Milli-Q water, the samples were investigated by means of TEM.

\subsection{Catalytic performances of Au nanoplates/GO composites toward the catalytic reduction of 4-NP}

The catalytic performances of our Au nanoplates/GO composites towards the reduction of 4 -NP by $\mathrm{NaBH}_{4}$ were carried out using a quartz cell ( $1 \mathrm{~cm}$ path length) as the reactor. In a typical process, $400 \mu \mathrm{L}$ aqueous solution of $4-\mathrm{NP}(1.4 \mathrm{mM})$ and $400 \mu \mathrm{L}$ freshly prepared aqueous solution of $\mathrm{NaBH}_{4}(0.08 \mathrm{M})$ were added to $1.8 \mathrm{~mL}$ Milli-Q water. Subsequently, $1 \mathrm{~mL}$ aqueous dispersion of our composites ( $c a .1 .2 \mathrm{mg}$ ) was added into this mixture under magnetic stirring, during which the real-time UV-vis spectra of the reaction system were measured to monitor the progress of the catalytic reaction. For the evaluation of the catalytic activities, $C$ is the concentration of 4-NP molecules at a real-time $t$, and $C_{0}$ is the concentration of 4-NP solution before the introduction of catalysts. Controlled experiments without the use of our composites were also conducted under the similar experimental conditions. All the operations were conducted under ambient conditions. 


\subsection{Apparatus and measurements}

The scanning electron microscopy (SEM) measurements were carried out using a Hitachi S-4800 system. The energy dispersive X-ray spectroscopy (EDX) was measured with a Horiba EMAX Xact energy dispersive spectrometer that was attached to the Hitachi S-4800 system. The transmission electron microscopy (TEM), high-resolution TEM (HRTEM), and the selective-area electron diffraction (SAED) patterns of the samples were measured with a JEM-2100F, which was operated with an accelerating voltage of $200 \mathrm{kV}$. A JASCO IR-660 spectrometer was employed for the FT-IR spectral measurements. The X-ray diffraction (XRD) measurements were performed on a PANalytical X'Pert PRO instrument with $\mathrm{Cu} \mathrm{K} \alpha$ radiation. The catalytic reduction of 4-NPs was monitored by measuring the realtime UV-vis spectra of the catalytic systems using a Hitachi U3900 spectrometer. The X-ray photoelectron spectroscopy (XPS) was performed on an ESCALab 220i-XL electron spectrometer from VG Scientific using $300 \mathrm{~W}$ Al $\mathrm{K} \alpha$ radiation. The binding energies were referenced to the $\mathrm{C} 1 \mathrm{~s}$ line at $284.8 \mathrm{eV}$ from adventitious carbon. The Raman spectra of our samples were recorded on a Renishaw inVia plus Raman microscope using a $514.5 \mathrm{~nm}$ argon ion laser. The zeta potential measurements of our GO aqueous solutions were performed using a Zetasizer Nano ZS ZEN3600 (Malvern) instrument. Except noted, all the measurements were conducted under ambient conditions.

\section{Results and discussion}

To prepare our $\mathrm{Au}$ nanoplates/GO composites, an aqueous solution of potassium tetrachloroaurate(III) was mixed with an aqueous dispersion of GO nanosheets and ethanol. The asobtained dispersion was aged under ambient conditions and the products were collected and washed fully with ultra-pure Milli-Q water by means of centrifugation. The morphology of the as-manufactured products was examined by scanning electron microscopy (SEM). As shown in Fig. 1a, platelike nanostructures with a hexagonal, truncated hexagonal, triangular or irregular morphology, most of which manifest themselves with an edge length of 100-400 $\mathrm{nm}$ and a thickness of $10-30 \mathrm{~nm}$, could be observed from the typical SEM image of the assynthesized samples. The components of the samples were investigated by energy dispersive X-ray spectroscopy (EDX) analysis. As shown in Fig. S1, $\uparrow \mathrm{C}$ and $\mathrm{O}$ elements of GO and Au
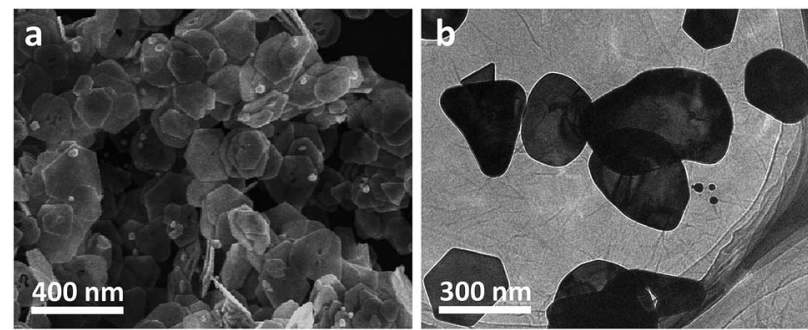

Fig. 1 The typical SEM (a) and low-resolution TEM (b) images of our Au nanoplates/GO composites. element could be detected distinctly. The atomic ratio of $\mathrm{C}: \mathrm{O}: \mathrm{Au}$ is evaluated to be $c a .1: 0.2: 0.26$. These results preliminarily indicate the formation of $\mathrm{Au}$ nanoplates/GO composites.

The morphology of thus-produced nanostructures has also been investigated by means of transmission electron microscopy (TEM). As shown in Fig. 1b, it can be seen that platelike nanostructures, whose dimensions and morphologies are nearly similar to the results of the SEM image (Fig. 1a), are observed from the representative low-resolution TEM (LRTEM) image of the samples. In addition, the presence of wrinkled GO nanosheets, on which Au nanoplates are distributed, could also be evidently observed. On the one hand, it should be noted that the as-synthesized nanostructures were collected and washed fully through centrifugation and resuspension treatments several times. On the other hand, we also found that when the aqueous of GO nanosheets were treated under the similar conditions, no precipitates could be observed from the bottom or the wall of the centrifuge tubes. This suggests that GO nanosheets dispersed in water could not be collected simply by a centrifugation under our experimental conditions. Together with the above-mentioned EDX and TEM results, these experimental facts further imply the formation of $\mathrm{Au}$ nanoplates/GO composites. ${ }^{\mathbf{4 7}, 48}$

This could be validated by a comparison of the Fourier transform infrared spectra (FT-IR) of the powdery GO nanosheets and the as-formulated samples. As shown in Fig. 2, it can be seen that the powdery GO nanosheets exhibit several characteristic bands around 1734, 1640, and $1437 \mathrm{~cm}^{-1}$, which could be attributed to the $\mathrm{C}=\mathrm{O}$ carbonyl stretching, the vibrations of the adsorbed water molecules, the skeletal vibrations of the unoxidized graphitic domains and the $\mathrm{O}-\mathrm{H}$ deformation vibration, respectively. ${ }^{\mathbf{4 6 9 , 5 0}}$ In the case of the as-synthesized nanostructures, the vibration of $\mathrm{C}=\mathrm{O}$ carbonyl stretching shifts to $1717 \mathrm{~cm}^{-1}$. Compared with that of the powdery GO nanosheets, this peak displays $c a .17 \mathrm{~cm}^{-1}$ shift to the lower wavenumber. Accompanied by the aforementioned EDX and

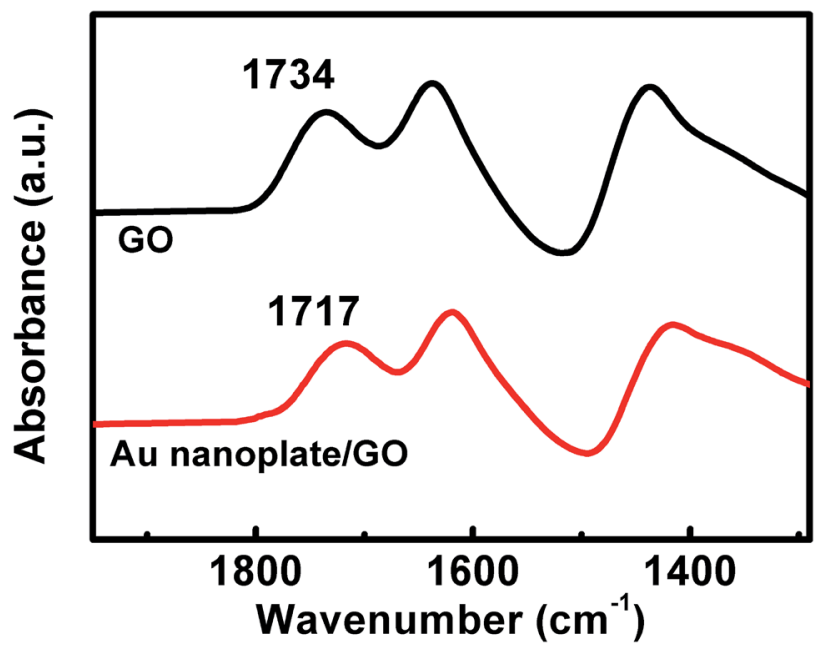

Fig. 2 The typical FT-IR spectra of the powdery GO and the asfabricated Au nanoplates/GO nanostructures. 
TEM results shown in Fig. $\mathrm{S} 1 \dagger$ and $1 \mathrm{~b}$, respectively, these facts verify the hybridization between our Au nanoplates and GO nanosheets. ${ }^{46,49-51}$

The fabrication of our Au nanoplates was achieved with the assistance of GO nanosheets. To elucidate the role of GO, controlled experiments without the use of GO nanosheets were also conducted under the similar experimental conditions. In this case, pure Milli-Q water but not an aqueous dispersion of GO was introduced into the synthesis system. As presented in Fig. S2, $\dagger$ the typical SEM image of the as-synthesized samples indicates that only irregular Au nanoparticles but not platelike nanostructures could be obtained. Considering the results shown in Fig. 1, wherein the samples were fabricated in the presence of GO nanosheets, these present results suggest that GO nanosheets are of critical significance for the manufacturing of our platelike Au nanostructures.

Structurally, GO is a chemically modified graphene sheet functionalized with various oxygen-containing groups on its basal plane and edges. ${ }^{34,35}$ These oxygen-containing groups confer GO with negative surface charges, ${ }^{52}$ and provide it with numerous active sites for the production of hybrid nanostructures. ${ }^{34}$ As validated by zeta potential measurements, our GO nanosheets are negatively charged with a zeta potential of ca. $-38.6 \mathrm{mV}$. On the other hand, as it is known, for the fabrication of GO- or oxygen groups containing polymers-hybridized $\mathrm{Au}$ nanostructures, the $\mathrm{AuCl}_{4}{ }^{-}$would interact with the oxyfunctional groups in terms of $\mathrm{Au}^{3+}$ cations through a replacement of $\mathrm{Cl}^{-}$ligands. ${ }^{53-55}$ Accordingly, a localized enrichment of $\mathrm{Au}^{3+}$ nearby the oxy-functional groups would occur via coordination, electrostatic or dipole interactions such that the oxy- functional groups would serve as implantation sites for the nucleation and growth of Au nanostructures during the ethanolinduced reduction of $\mathrm{Au}^{3+}{ }^{33,54,56}$

As illustrated in Fig. 3, the evolution behaviours of our $\mathrm{Au}$ nanoplates during the synthesis were investigated by measuring the TEM images of the samples generated at a desired aging time. It can be seen that an aging time of 0 minute only gives out GO nanosheets, on which no nanoparticles could be observed (Fig. 3a). On the other hand, translucent GO nanosheets decorated with tiny nanoparticles of a size of $c a .3 \mathrm{~nm}$ are obtained when the aging time is 30 minutes (Fig. $3 \mathrm{~b}$ ). It should be noted that most of the nanoparticles are anchored on the surface of GO nanosheets, while nearly no free gold nanoparticles, which stand away from the GO nanosheets could be perceived. Together with the above-addressed concise backgrounds of GO, these observations indicate the initiation of Au nucleation, wherein the oxy-functional groups of GO nanosheets work as implantation sites. ${ }^{53,54,56}$ Moreover, as shown in Fig. 3c, when the evolution of the Au nanoparticles was terminated at an aging time of 60 minutes, the number of the tiny nanoparticles formed at the initial stage displays an evident decrease, whereas relatively bigger platelike nanostructures with a size of $c a$. $15 \mathrm{~nm}$ came into being.

When the aging time was extended to 12 hours (Fig. 3d), the nanostructures formed in the previous stage become bigger with a sized of $c a .20-50 \mathrm{~nm}$ and exhibit a triangular or hexagonal morphology. At the same time, some tiny nanostructures with a size of $c a .3 \mathrm{~nm}$ were observed at this stage again. Moreover, when the aging time was further prolonged to 3 days (Fig. 3e), the triangular or hexagonal nanostructures became
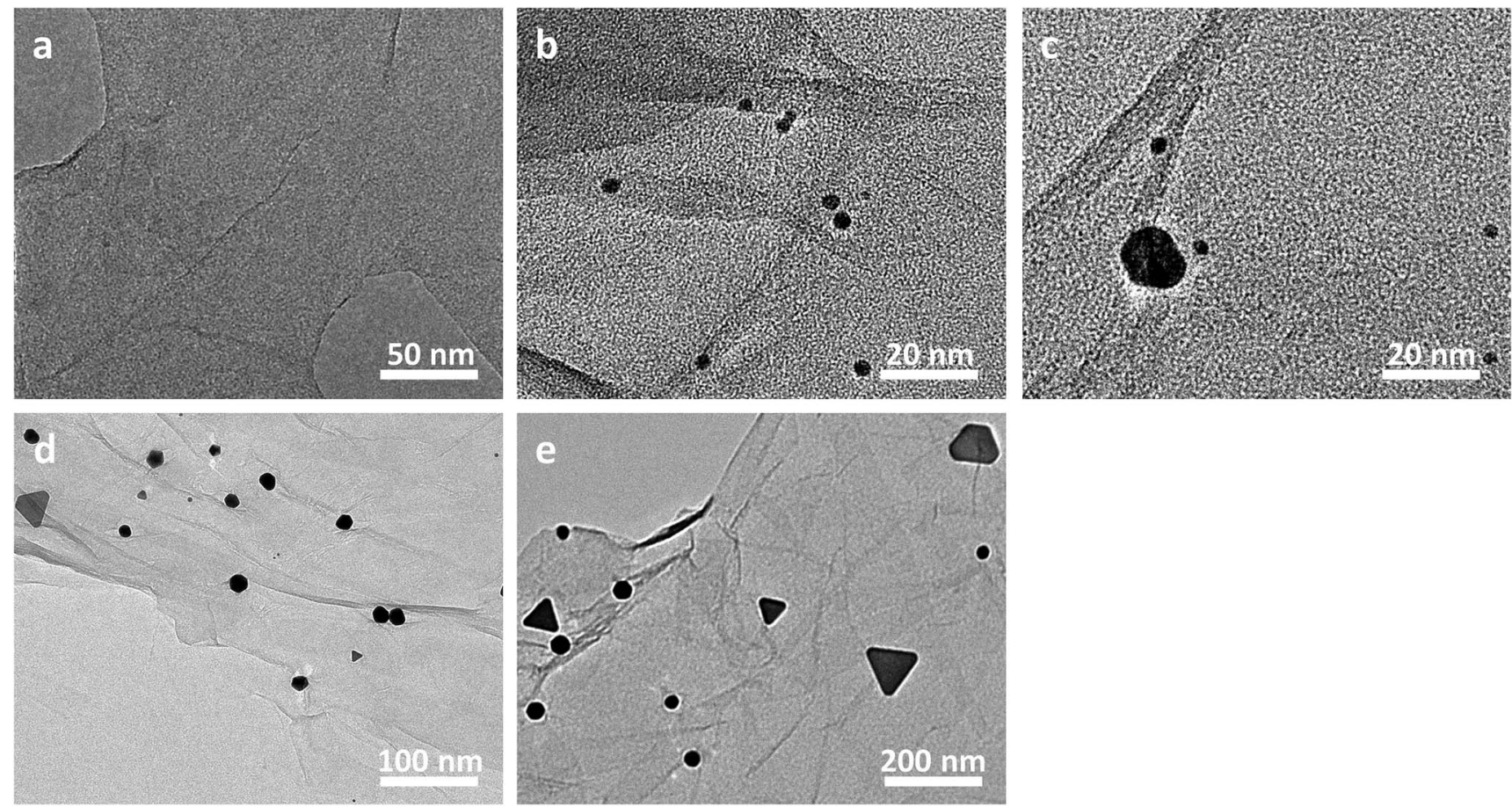

Fig. 3 The real-time TEM images of the as-fabricated nanostructures generated at a desired aging stage. The aging time is 0 minute (a), 30 minutes (b), 60 minutes (c), 12 hours (d), 3 days (e), respectively. 
even bigger with a size of $c a$. 50-100 $\mathrm{nm}$. Interestingly, a redisappearance of the tiny nanospecies of $c a .3 \mathrm{~nm}$ is observed at this stage. Finally, nanoplates of a triangular, hexagonal, truncated hexagonal or irregular morphology, which display a dimension of $c a$. 100-400 $\mathrm{nm}$ are obtained when the aging time was extended to 10 days, as shown in Fig. 1b. These experimental results suggest that our $\mathrm{Au}$ nanoplates might be formed via a simultaneous ethanol-induced $\mathrm{Au}^{3+}$ reduction ${ }^{57}$ and Ostwald ripening (disappearance of the tiny nanospecies accompanied by a growth of the bigger ones) process. ${ }^{53}$

To disclose the crystalline structure of our $\mathrm{Au}$ nanoplates, their HRTEM images and the corresponding SAED patterns were measured. The measurements were conducted with the incident electron beam perpendicular to the basal plane of the Au nanoplates. As shown in Fig. 4, distinct lattice fringes, which indicate an interplane spacing of 0.25 and $0.14 \mathrm{~nm}$, could be observed from the HRTEM image. These values are in good agreement with the interplane spacing of the 1/3(422) and $(-2-20)$ facets of $\mathrm{Au}$ crystal, respectively, which has a facecentred cubic (fcc) structure. ${ }^{58}$ In the case of the corresponding SAED patterns, hexagonally arranged diffraction spots, which could be indexed to the 1/3(422) and (-2-20) facets of fcc $\mathrm{Au}$ crystal and indicate an interlattice spacing of 0.25 and $0.14 \mathrm{~nm}$, respectively, could be observed. These HRTEM and SAED results indicate that the basal plane of our Au nanoplates are enclosed by (111) crystal facets. ${ }^{58}$ We note that similar HRTEM and SAED results are obtained from the different sites of the same sample, suggesting a good single crystalline nature of our Au nanoplates.

The crystal structure of our samples was also examined by powder X-ray diffraction (PXRD), as shown in Fig. 5. It can be seen that the PXRD curve exhibits evident diffraction peaks at ca. $38.2^{\circ}, 44.4^{\circ}, 64.6^{\circ}, 77.6^{\circ}$ and $81.7^{\circ}$, which could be indexed well to the (111), (200), (220), (311), and (222) facets of the typical fcc phase of Au (JCPDS no. 04-0784), respectively. ${ }^{59-61}$ Meanwhile, negligible diffraction peaks that attributed to GO nanosheets could be observed from the PXRD curve. This could

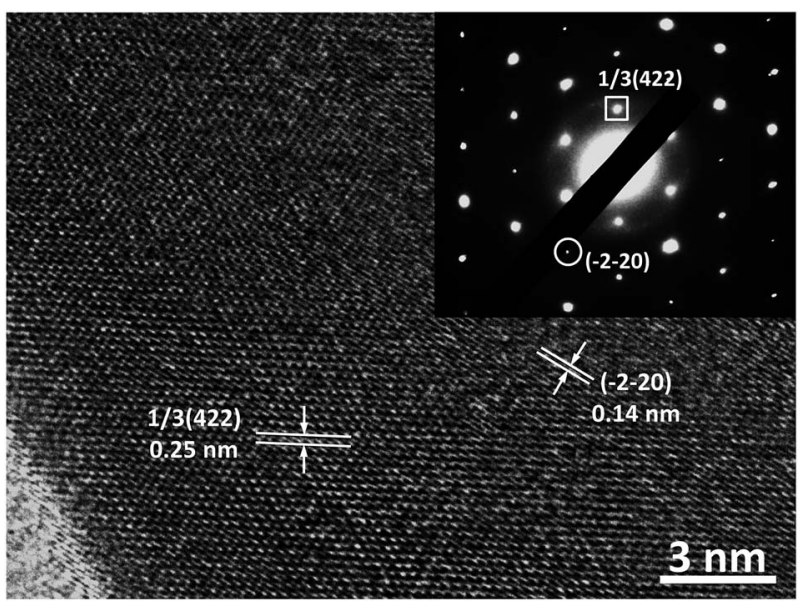

Fig. 4 The typical HRTEM image and the corresponding SAED pattern of our nanoplates obtained with the incident electron beam perpendicular to the basal plane of the nanoplates.

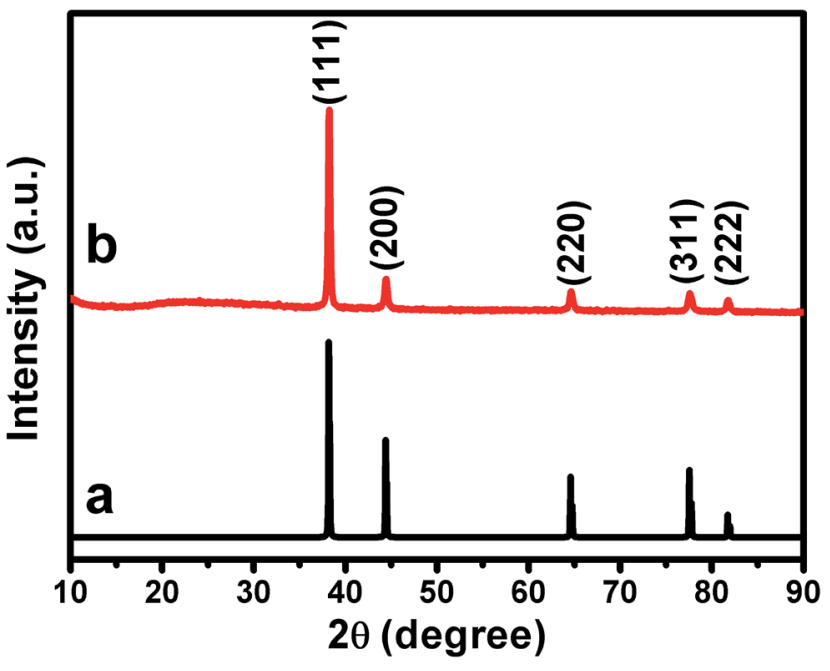

Fig. 5 The XRD pattern of (a) the standard JCPDS file of fcc phase of Au crystal (JCPDS no. 04-0784), and (b) the as-prepared Au nanoplate/ GO composites.

be owing to the low diffraction intensity of GO. ${ }^{62}$ Remarkably, it is noted that the intensity ratio of the (200), (220) and (311) peaks to the (111) peak is evaluated to be $c a .0 .18,0.11$ and 0.10 , respectively. These values are less than $1 / 3$ and are substantially lower than those of the corresponding data of the standard JCPDS file of Au (JCPDS no. 04-0784), which is generally ca. 0.51, 0.32 and 0.36 , respectively. Accompanied by the results of HRTEM image and SAED pattern shown in Fig. 4, the observation of this extraordinary prominent (111) peak further confirms that our $\mathrm{Au}$ nanoplates are single crystals with (111) facets as their basal plane. The UV-vis spectra of our Au/GO composites were also measured. As shown in Fig. S3, $\dagger$ it can be seen that distinct absorptions in the range of 500-600 nm could be observed. This is owing to the production of metallic $\mathrm{Au}$ nanostructures, which could arouse surface plasmon resonance (SPR) absorptions in the visible range. ${ }^{3}$

As known, 4-aminophenol (4-AP) is an industrially important chemical broadly used in various fields, such as photographic developer, corrosion inhibitor, analgesic and antipyretic drugs, anticorrosion lubricant, etc. ${ }^{\mathbf{6 3 , 6 4}}$ In the presence of noble metals, the catalytic reduction of 4-nitrophenol (4-NP) using $\mathrm{NaBH}_{4}$ as reducing reagent is considered to be one of the most frequently employed approaches for the manufacture of 4-AP. ${ }^{59,63,64}$ On the other hand, it has been reported that $\mathrm{Au}$ nanostructures predominately exposed with (111) facets could work as excellent catalysts for the reduction of $4-\mathrm{NP}$ by $\mathrm{NaBH}_{4} \cdot{ }^{53,59}$ This is because that, among the crystal planes of fcc crystals, the atomic arrangement in the (111) facet has the highest atomic planar density. ${ }^{53,59,65}$ As proved in the above sections, the basal plane of our $\mathrm{Au}$ nanoplates is enclosed by the (111) facet. This might endow our $\mathrm{Au}$ nanoplates/GO composites with excellent catalytic performances towards the reduction of 4-NP.

Motivated by these issues, the catalytic behaviors of our composites toward the reduction of 4-NPs were investigated, wherein the catalytic performances were monitored by measuring the real-time UV-vis spectra of the reaction system. 
As shown in Fig. 6a and $\mathrm{S} 4, \dagger$ when the reaction was conducted without the addition of our composites, negligible changes could be observed from the UV-vis spectra. These results suggests an inefficient reduction of $4-\mathrm{NP}$ by $\mathrm{NaBH}_{4}$ under our experimental conditions. On the other hand, as illustrated in Fig. S5, $\uparrow$ upon the addition of our composites into the reaction system under the similar experimental conditions, a continuous decrease is observed from the absorption peak at $c a$. $400 \mathrm{~nm}$. At the same time, a concomitant increase of a new absorption at $c a .300 \mathrm{~nm}$ is also detected. These observations indicate that our composites could work as catalysts towards the reduction of 4-NPs to 4-APs. ${ }^{59,66}$ However, it is found that the UV-vis spectra of the system exhibit only trivial decrease at $400 \mathrm{~nm}$ and slight increase at $300 \mathrm{~nm}$ within the first 12 minute of the catalytic reaction (Fig. S5†), during which only ca. 11\% 4NPs were reduced (Fig. 6a). As plotted in Fig. 6b, a good linear correlation between reaction time $(t)$ and $\ln \left(C / C_{0}\right)$ could be observed from this period. This indicates that the reduction of 4-NPs over our composites proceeds with first-order kinetics $-\mathrm{d} C / \mathrm{d} t=k C$, in which $k$ is the rate constant of the reaction. Different as what we have expected, a rate constant of as low as
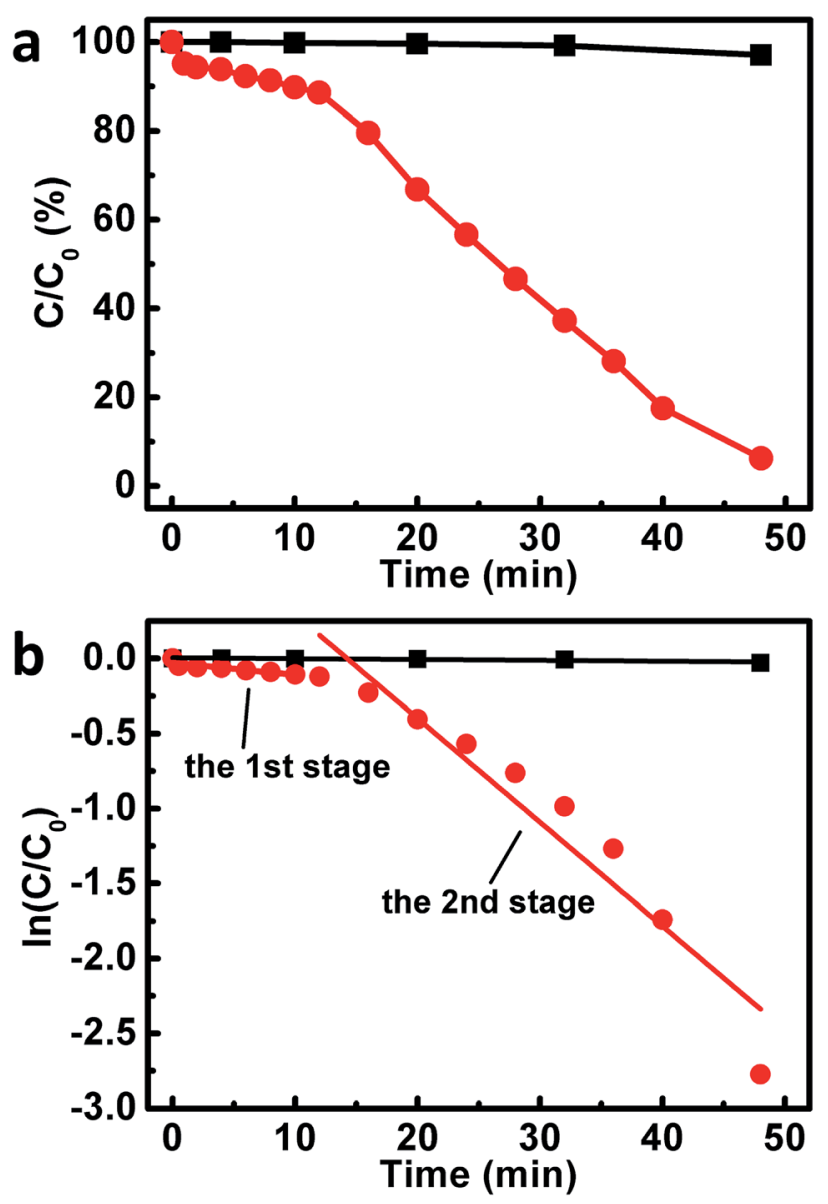

Fig. 6 The catalytic performances (a) and the corresponding kinetic linear simulation curves (b) of our catalysts towards the reduction of 4NP. Black curves: the results of a blank experiment, wherein no catalysts are used. Red curves: the reaction is conducted in the presence of our Au nanoplates/GO nanocomposites. ca. $8.2 \times 10^{-3} \mathrm{~min}^{-1}$ could be derived at this stage, although the basal planes of our Au nanoplates are enclosed by (111) facets, which have been verified to be highly catalytically active facets. ${ }^{53,59}$

Fascinatingly, it is found that, starting from the 12th minute after the addition of our composites into the reaction system, the absorption peak at $c a .400$ and $300 \mathrm{~nm}$ decreases and increases rapidly, respectively (Fig. S5†). Nearly 94\% 4-NPs are reduced when the reaction proceeds for another 36 minutes (Fig. 6a). At this stage, a rate constant of ca. $7.0 \times 10^{-2} \mathrm{~min}^{-1}$ could be obtained, as shown in Fig. $6 \mathrm{~b}$. These results indicate an interesting two-stage catalytic behavior of our Au nanoplate/ GO composites, wherein the catalytic activity of the second stage displays a substantial enhancement by a factor of $c a .9$ times compared to that of the initial stage.

To elucidate such fascinating two-stage catalytic performances, the Raman spectra of our original composites, and those of the samples, which have been used as catalysts in the reaction systems for 12 and 48 minutes (defined as usedcomposites-12 and used-composites-48, respectively, hereafter), are examined. As shown in Fig. 7 (black curve), it can be seen that a D-band at $c a .1348 \mathrm{~cm}^{-1}$ and a G-band around $1600 \mathrm{~cm}^{-1}$ could be observed from the original composites. The relative intensity of the D-band is lower than that of the G-band with an intensity ratio of $c a . I_{\mathrm{D}} / I_{\mathrm{G}}=0.69$. These are typical Raman characteristics of GO nanosheets. ${ }^{67,68}$ In contrast, for the Raman spectrum of the used-composites-12, the relative intensity of D-band is higher than that of the G-band with an intensity ratio of $c a . I_{\mathrm{D}} / I_{\mathrm{G}}=1.21$ (Fig. 7, red curve). Nearly similar observations are obtained from that of the usedcomposites-48 (Fig. 7, blue curve). These results indicate the reduction of $\mathrm{GO}$ by $\mathrm{NaBH}_{4}$ during the first stage of the catalytic reactions, ${ }^{69}$ leading to the formation of reduced (RGO) nanosheets of partially restored $\pi$-conjugated aromatic network. ${ }^{70}$

As known, a charge transfer generally occurs between GO and the hybridized components, wherein the G-band of GO

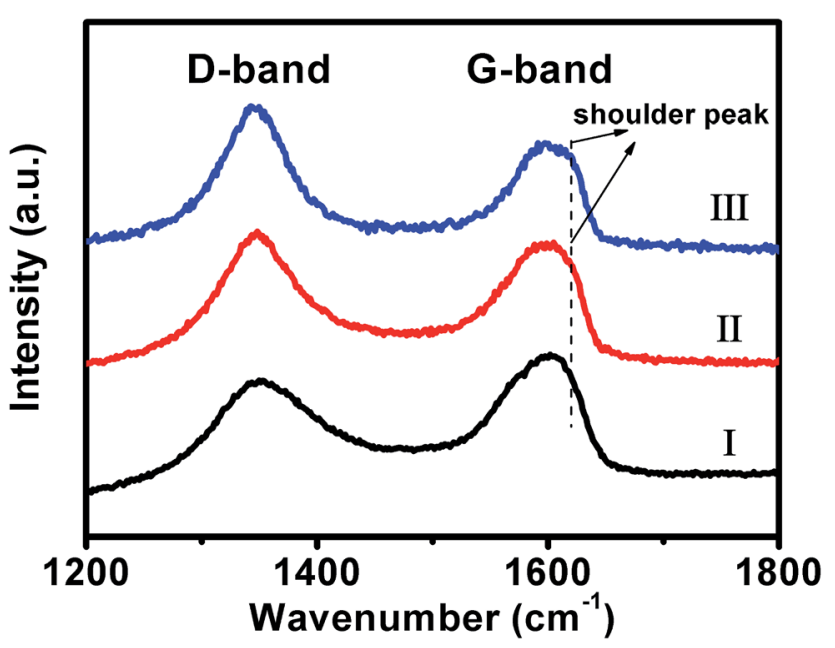

Fig. 7 The typical Raman spectra of our original Au nanoplate/GO composites (I), the used-composites-12 (II), and used-composites-48 (III). 
shifts to higher frequency when an electron acceptor component is hybridized, while it shifts to lower frequency upon a hybridization of an electron donor component. ${ }^{46,71-73}$ In our present case, besides the above-mentioned characteristics, a shoulder G-band around $c a .1620 \mathrm{~cm}^{-1}$, which shifts by $c a$. $20 \mathrm{~cm}^{-1}$ to higher frequency, could also be observed from the Raman spectra of the used-composites- 12 and used-composites48 (Fig. 7, red and blue curves). This result suggests that, the reduction of GO nanosheets by $\mathrm{NaBH}_{4}$ in the initial stage of the catalytic reduction of 4-NPs, which results in a partial restoration of the $\pi$-conjugated aromatic network of GO, promotes an electron transfer from GO nanosheets to Au nanoplates. ${ }^{46,71-73}$ It has been demonstrated that the electron transfer from GO nanosheets to $\mathrm{Au}$ nanostructures could increase the local electron concentration and thus facilitate the uptake of electrons by 4-NP molecules, leading to an enhanced catalytic performances. ${ }^{74-76}$ Together with the above-described results of Raman spectra, we suggest that the $\mathrm{NaBH}_{4}$-induced reduction of GO at the initial stage of the catalytic performances, wherein the $\pi$-conjugated aromatic structure of GO has been partially repaired and the electron transfer from GO to Au nanoplates has accordingly been promoted, might contribute to the substantially boosted catalytic reactivity of our composites observed at the second stage.

To verify the above proposals, the X-ray photoelectron spectroscopy (XPS) of our composites of different catalytic stages was examined. As shown in Fig. 8a, the C 1s XPS spectra of our original composites before the catalytic uses display evident $\mathrm{C}-\mathrm{C}(284.8 \mathrm{eV}), \mathrm{C}-\mathrm{O}(286.8 \mathrm{eV}), \mathrm{C}=\mathrm{O}(287.6 \mathrm{eV})$ and $\mathrm{C}(\mathrm{O})-\mathrm{O}$ $(289.2 \mathrm{eV})$ peaks, which are typical XPS spectral features of GO nanosheets. ${ }^{76}$ The atom ratio between oxygen and carbon (O/C) in this case is evaluated to be $c a$. 0.47 . For the used-composites12 and used-composites-48, the peaks ascribing to the oxyfunctional groups display a substantial decrease, wherein the $\mathrm{O} / \mathrm{C}$ is evaluated to be $c a$. 0.07 . This value is evidently lower than the corresponding data of the original composites, further confirming the $\mathrm{NaBH}_{4}$-induced reduction of $\mathrm{GO}$ nanosheets during the initial stage of the catalytic performances. Meanwhile, as shown in Fig. 8b, the Au 4 XPS spectra of the original composites exhibit a doublet from the spin orbital splitting of $4 \mathrm{f}_{7 / 2}$ and $4 \mathrm{f}_{5 / 2}$ states, which locate at $c a .84 .6 \mathrm{eV}$ and $88.3 \mathrm{eV}$, respectively. ${ }^{77}$ In the cases of the used-composites-12 and usedcomposites-48, these peaks manifest themselves at $c a .84 .1 \mathrm{eV}$ and $87.8 \mathrm{eV}$, which exhibit nearly $0.5 \mathrm{eV}$ shift to lower binding energy compared to those of the original composites before the reduction of GO. Accompanied by the results of Raman spectra shown in Fig. 7, these experimental facts solidly verify that the $\mathrm{NaBH}_{4}$-induced partial restoration of the $\pi$-conjugated aromatic network of GO occurred during the first stage could indeed facilitate electron transfer from GO to Au nanoplates. ${ }^{46,78}$ This favors an increase of the local electron concentration and the uptake of electrons by 4-NP molecules, ${ }^{74-76}$ resulting in the evidently enhanced catalytic performance of the second stage (Fig. 6).

To further confirm the above-mentioned proposals, a controlled experiment has been conducted under the similar experimental conditions except that the $\mathrm{Au} / \mathrm{GO}$ composite was
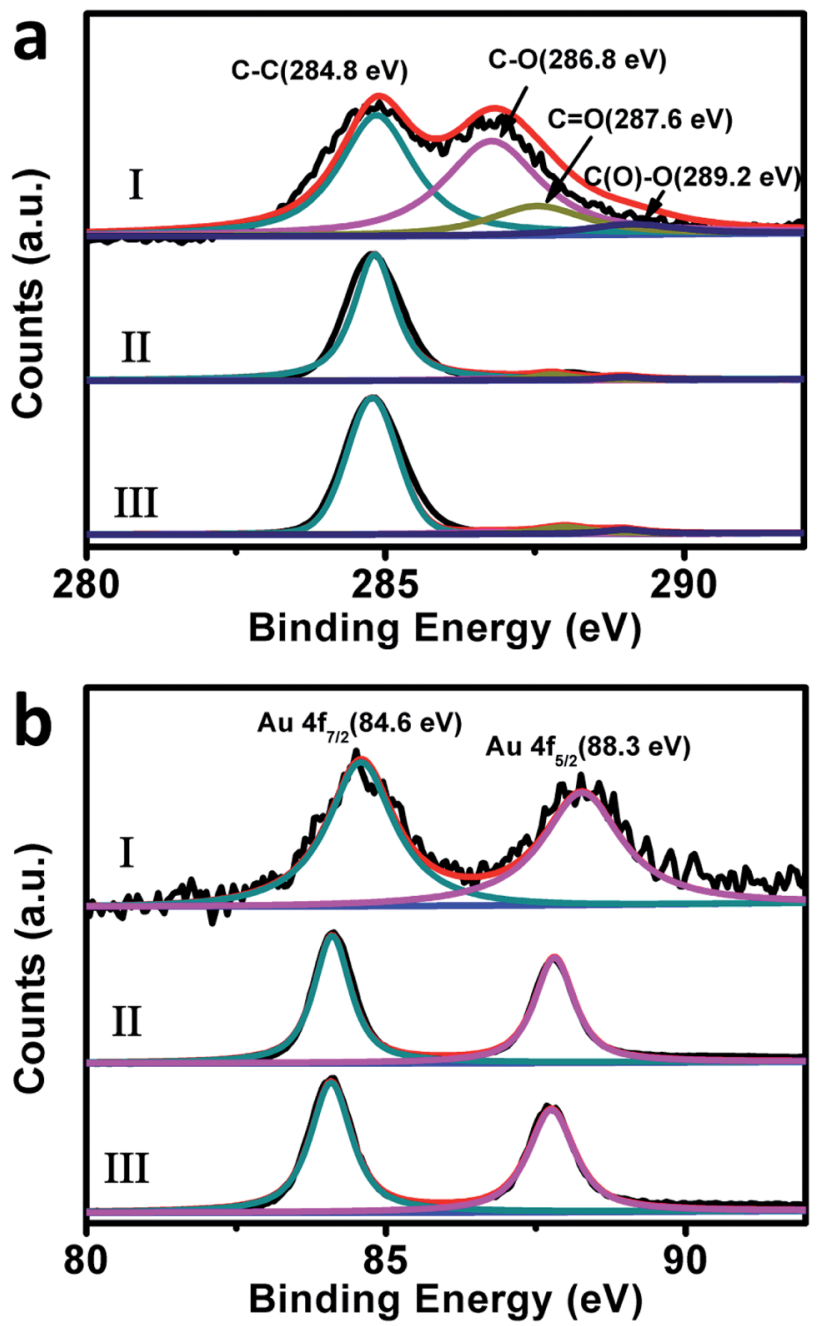

Fig. 8 The $C$ 1s (a) and Au $4 f$ (b) XPS spectra of our original Au nanoplate/GO composites (I), the used-composites-12 (II), and usedcomposites-48 (III).

reduced by $\mathrm{NaBH}_{4}$ firstly, after which they are used as catalysts towards the reduction of 4-NP. As shown in Fig. S6, $\dagger$ instead of a two-stage catalytic behavior, ca. $98.8 \%$ 4-NPs was reduced directly within 28 minutes. As plotted in Fig. S6c, $\dagger$ a good linear correlation between reaction time $(t)$ and $\ln \left(C / C_{0}\right)$ could be observed, giving out a rate constant of $c a .15 \times 10^{-2} \mathrm{~min}^{-1}$. This value is in the same order as that observed from the second stage of the above-discussed catalytic performances, confirming our proposals.

As proved by Raman (Fig. 7) and XPS spectra (Fig. 8), a reduction of GO nanosheets occurs within the first stage of the catalytic performances, resulting in the formation of RGO nanosheets. As it is widely known, compared to GO nanosheets of abundant $\mathrm{sp}^{3}$-hybridized carbon atoms, RGO nanosheets consist of much more $\mathrm{sp}^{2}$-hybridized carbon atoms, leading to a partially restored $\pi$-conjugated aromatic structure. ${ }^{70}$ This makes RGO nanosheets good scaffolds for anchoring $\pi$-conjugated molecules via $\pi-\pi$ stacking interactions. ${ }^{79,80}$ We suggest that the RGO-facilitated anchoring of 4-NP molecules to our composites might also contribute partially to the substantially 
enhanced catalytic performances observed from the second stage. Similar proposals have previously been reported by others. $^{74}$

Finally, the SEM image (Fig. S7a $\dagger$ ) and HRTEM pattern (Fig. S7b $\dagger$ ) of our composites after catalytic performances have been investigated. It can be seen that compared to the corresponding results of the original composites before the catalytic uses (Fig. 1a and 4), negligible changes could be observed from the samples. Accompanied by the discussions presented in the above paragraphs, these experimental facts further suggest that the enhanced catalytic performances observed from the second stage are mainly aroused by the reduction of GO species but not by the changes in the morphology and the crystalline structure of our $\mathrm{Au} / \mathrm{GO}$ composites.

\section{Conclusions}

In summary, we herein report that GO-hybridized Au nanoplates could be easily fabricated with the assistance of GO nanosheets under ambient conditions. In our new fabrication protocol, no non-environmentally friendly chemicals, rigorous experimental conditions, or specific apparatus, etc. are essentially required. We show that the as-formulated Au nanoplates/ GO composites could work as catalysts to towards the $\mathrm{NaBH}_{4}{ }^{-}$ induced reduction of 4-NP molecules, wherein an interesting two-stage catalytic behaviour has been observed. Compared with that of the first stage, the catalytic reactivity of the second stage exhibits a substantial enhancement by a factor of $c a .9$ times. It is disclosed that the boosted catalytic performances could be ascribed to the $\mathrm{NaBH}_{4}$-induced restoration of the $\pi$ conjugated aromatic network of GO. We propose that the asrestored $\pi$-conjugated aromatic structures, which promote electron transfer from GO nanosheets to Au nanoplates and favor an anchoring of 4-NP molecules to our catalysts, might contribute much to the boosted catalytic performances. On the one hand, our investigation likely provides a facile, low cost yet environmentally benign method for a one-pot production of $\mathrm{Au}$ nanoplates. On the other hand, with regard to an intriguing two-stage catalytic performance, our work might offer deep scientific insights into the GO-hybridized advanced catalysts, which is an issue of paramount importance and broad concern.

\section{Conflicts of interest}

There are no conflicts to declare.

\section{Acknowledgements}

We acknowledge the financial support from the National Natural Science Foundation of China (Grants 21673253, 21372225, 20873159, 21321063, and 91027042), the National Key Basic Research Project of China (Grants 2013CB834504 and 2011CB932301), and the Chinese Academy of Sciences (Grants XDA09030200, XDB12020200 and 1731300500015).

\section{Notes and references}

1 J. Xiao and L. Qi, Nanoscale, 2011, 3, 1383-1396.

2 L. Qi, Coord. Chem. Rev., 2010, 254, 1054-1071.

3 N. Li, P. Zhao and D. Astruc, Angew. Chem., Int. Ed., 2014, 53, 1756-1789.

4 P. Priecel, H. A. Salami, R. H. Padilla, Z. Zhong and J. A. Lopez-Sanchez, Chin. J. Catal., 2016, 37, 1619-1650.

5 W. Wang, J. Gu, W. Hua, X. Jia and K. Xi, Chem. Commun., 2014, 50, 8889-8891.

6 Y. Zhang, G. Chang, S. Liu, W. Lu, J. Tian and X. Sun, Biosens. Bioelectron., 2011, 28, 344-348.

7 R. Ning, W. Lu, Y. Zhang, X. Qin, Y. Luo, J. Hu, A. M. Asiri, A. O. Al-Youbi and X. Sun, Electrochim. Acta, 2012, 60, 13-16.

8 B. Cao, B. Liu and J. Yang, CrystEngComm, 2013, 15, 57355738.

9 L. Scarabelli, M. Coronado-Puchau, J. J. Giner-Casares, J. Langer and L. M. Liz-Marzán, ACS Nano, 2014, 8, 58335842.

10 W.-H. Lin, Y.-H. Lu and Y.-J. Hsu, J. Colloid Interface Sci., 2014, 418, 87-94.

11 Z. Yang, Z. Li, X. Lu, F. He, X. Zhu, Y. Ma, R. He, F. Gao, W. Ni and Y. Yi, Nano-Micro Lett., 2017, 9, 5.

12 B. Seo, S. Choi and J. Kim, ACS Appl. Mater. Interfaces, 2011, 3, 441-446.

13 P. Pienpinijtham, X. X. Han, T. Suzuki, C. Thammacharoen, S. Ekgasit and Y. Ozaki, Phys. Chem. Chem. Phys., 2012, 14, 9636-9641.

14 Y. Huang, A. R. Ferhan, Y. Gao, A. Dandapat and D.-H. Kim, Nanoscale, 2014, 6, 6496-6500.

15 M. B. E. Griffiths, S. E. Koponen, D. J. Mandia, J. F. McLeod, J. P. Coyle, J. J. Sims, J. B. Giorgi, E. R. Sirianni, G. P. A. Yap and S. T. Barry, Chem. Mater., 2015, 27, 6116-6124.

16 S. Balasubramanian, S. R. Bezawada and D. Raghavachari, ACS Sustainable Chem. Eng., 2016, 4, 3830-3839.

17 Y. Shao, Y. Jin and S. Dong, Chem. Commun., 2004, 11041105.

18 Y. N. Tan, J. Y. Lee and D. I. C. Wang, J. Phys. Chem. C, 2009, 113, 10887-10895.

19 S. Yang, Y. Wang, Q. Wang, R. Zhang, Z. Yang, Y. Guo and B. Ding, Cryst. Growth Des., 2007, 7, 2258-2261.

20 C. S. Ah, Y. J. Yun, H. J. Park, W.-J. Kim, D. H. Ha and W. S. Yun, Chem. Mater., 2005, 17, 5558-5561.

21 T. Deckert-Gaudig and V. Deckert, Small, 2009, 5, 432-436. 22 T. Yu, Z. Wu and W.-S. Kim, RSC Adv., 2014, 4, 37516-37521. 23 J. Cao, S. Mei, H. Jia, A. Ott, M. Ballauff and Y. Lu, Langmuir, 2015, 31, 9483-9491.

24 G. Wang, S. Tao, Y. Liu, L. Guo, G. Qin, K. Ijiro, M. Maeda and Y. Yin, Chem. Commun., 2016, 52, 398-401.

25 L. Chen, H. Hu, Q. Liu, F. Ji, S. Chen, Y. Xu and Q. Zhang, J. Mater. Chem. C, 2016, 4, 6457-6460.

26 W. Xin, J.-M. Yang, C. Li, M. S. Goorsky, L. Carlson and I. M. De Rosa, ACS Appl. Mater. Interfaces, 2017, 9, 62466254.

27 Z. Guo, Y. Zhang, A. Xu, M. Wang, L. Huang, K. Xu and N. Gu, J. Phys. Chem. C, 2008, 112, 12638-12645. 
28 A. Miranda, E. Malheiro, E. Skiba, P. Quaresma, P. A. Carvalho, P. Eaton, B. de Castro, J. A. Shelnutt and E. Pereira, Nanoscale, 2010, 2, 2209-2216.

29 X. Sun, S. Dong and E. Wang, Angew. Chem., 2004, 116, 65206523.

30 Z. Wu, C. Dong, Y. Li, H. Hao, H. Zhang, Z. Lu and B. Yang, Angew. Chem., Int. Ed., 2013, 52, 9952-9955.

31 S. Kajimoto, D. Shirasawa, N. N. Horimoto and H. Fukumura, Langmuir, 2013, 29, 5889-5895.

32 L. Chen, F. Ji, Y. Xu, L. He, Y. Mi, F. Bao, B. Sun, X. Zhang and Q. Zhang, Nano Lett., 2014, 14, 7201-7206.

33 B. Tangeysh, K. M. Tibbetts, J. H. Odhner, B. B. Wayland and R. J. Levis, Nano Lett., 2015, 15, 3377-3382.

34 D. R. Dreyer, S. Park, C. W. Bielawski and R. S. Ruoff, Chem. Soc. Rev., 2010, 39, 228-240.

35 D. Chen, H. Feng and J. Li, Chem. Rev., 2012, 112, 6027-6053. 36 X. Huang, S. Li, Y. Huang, S. Wu, X. Zhou, S. Li, C. L. Gan, F. Boey, C. A. Mirkin and H. Zhang, Nat. Commun., 2011, 2, 292.

37 X. Huang, H. Li, S. Li, S. Wu, F. Boey, J. Ma and H. Zhang, Angew. Chem., Int. Ed., 2011, 50, 12245-12248.

38 J. Wang, G. Zhang, W. Sun, J. Sun, L. Luo, Z. Chang and X. Sun, Chem.-Eur. J., 2016, 22, 10923-10929.

39 Y. Zhang, S. Liu, W. Lu, L. Wang, J. Tian and X. Sun, Catal. Sci. Technol., 2011, 1, 1142-1144.

40 S. Yang, P. Qiu and G. Yang, Carbon, 2014, 77, 1123-1131.

41 J. Wang, X. Dong, R. Xu, S. Li, P. Chen and M. B. Chan-Park, Nanoscale, 2012, 4, 3055-3059.

42 X. Lou, H. Pan, S. Zhu, C. Zhu, Y. Liao, Y. Li, D. Zhang and Z. Chen, Catal. Commun., 2015, 69, 43-47.

43 L. Zhong, S. Gan, X. Fu, F. Li, D. Han, L. Guo and L. Niu, Electrochim. Acta, 2013, 89, 222-228.

44 W. S. Hummers and R. E. Offeman, J. Am. Chem. Soc., 1958, 80, 1339.

45 C. Nethravathi and M. Rajamathi, Carbon, 2008, 46, 19941998.

46 M. Zhu, P. Chen and M. Liu, ACS Nano, 2011, 5, 4529-4536.

47 G. Geng, B. Guan, P. Chen, M. Zhu, C. Yang and M. Liu, RSC Adv., 2017, 7, 9948-9957.

48 X. Zhang, M. Zhu, P. Chen, Y. Li, H. Liu, Y. Li and M. Liu, Phys. Chem. Chem. Phys., 2015, 17, 1217-1225.

49 D.-D. Zhang, S.-Z. Zu and B.-H. Han, Carbon, 2009, 47, 29933000.

50 G. I. Titelman, V. Gelman, S. Bron, R. L. Khalfin, Y. Cohen and H. Bianco-Peled, Carbon, 2005, 43, 641-649.

51 J. Petroski and M. A. El-Sayed, J. Phys. Chem. A, 2003, 107, 8371-8375.

52 M. Zhu, P. Chen and M. Liu, Langmuir, 2013, 29, 9259-9268.

53 M. Zhu, B. Lei, F. Ren, P. Chen, Y. Shen, B. Guan, Y. Du, T. Li and M. Liu, Sci. Rep., 2014, 4, 5359.

54 H. Yin, H. Tang, D. Wang, Y. Gao and Z. Tang, ACS Nano, 2012, 6, 8288-8297.

55 P. Tripathy, S. Ram and H. J. Fecht, Plasmonics, 2006, 1, 121127.

56 K. Jasuja and V. Berry, ACS Nano, 2009, 3, 2358-2366.
57 X. Wang, J. Zhuang, Q. Peng and Y. Li, Nature, 2005, 437, 121-124.

58 M. Yamamoto, Y. Kashiwagi, T. Sakata, H. Mori and M. Nakamoto, Chem. Mater., 2005, 17, 5391-5393.

59 B. Ballarin, M. C. Cassani, D. Tonelli, E. Boanini, S. Albonetti, M. Blosi and M. Gazzano, J. Phys. Chem. C, 2010, 114, 9693-9701.

60 T.-H. Lin, C.-W. Lin, H.-H. Liu, J.-T. Sheu and W.-H. Hung, Chem. Commun., 2011, 47, 2044-2046.

61 X. Huang, Y. Li, Y. Chen, E. Zhou, Y. Xu, H. Zhou, X. Duan and Y. Huang, Angew. Chem., 2013, 125, 2580-2584.

62 M. Zhu, P. Chen and M. Liu, Langmuir, 2012, 28, 3385-3390.

63 S. Saha, A. Pal, S. Kundu, S. Basu and T. Pal, Langmuir, 2010, 26, 2885-2893.

64 P. Hervés, M. Pérez-Lorenzo, L. M. Liz-Marzán, J. Dzubiella, Y. Lu and M. Ballauff, Chem. Soc. Rev., 2012, 41, 5577-5587. 65 E. Ghali, Corrosion Resistance of Aluminum and Magnesium Alloys: Understanding, Performance, and Testing, John Wiley \& Sons, Inc, Hoboken, New Jersey, 2010.

66 C. Wang, N. M. Markovic and V. R. Stamenkovic, ACS Catal., 2012, 2, 891-898.

67 S. Stankovich, D. A. Dikin, R. D. Piner, K. A. Kohlhaas, A. Kleinhammes, Y. Jia, Y. Wu, S. T. Nguyen and R. S. Ruoff, Carbon, 2007, 45, 1558-1565.

68 D. Yang, A. Velamakanni, G. Bozoklu, S. Park, M. Stoller, R. D. Piner, S. Stankovich, I. Jung, D. A. Field, C. A. Ventrice Jr and R. S. Ruoff, Carbon, 2009, 47, 145-152.

69 H.-J. Shin, K. K. Kim, A. Benayad, S.-M. Yoon, H. K. Park, I.-S. Jung, M. H. Jin, H.-K. Jeong, J. M. Kim, J.-Y. Choi and Y. H. Lee, Adv. Funct. Mater., 2009, 19, 1987-1992.

70 P. Yao, P. Chen, L. Jiang, H. Zhao, H. Zhu, D. Zhou, W. Hu, B.-H. Han and M. Liu, Adv. Mater., 2010, 22, 5008-5012.

71 A. Das, S. Pisana, B. Chakraborty, S. Piscanec, S. K. Saha, U. V. Waghmare, K. S. Novoselov, H. R. Krishnamurthy, A. K. Geim, A. C. Ferrari and A. K. Sood, Nat. Nanotechnol., 2008, 3, 210-215.

72 Y. Xu, Q. Wu, Y. Sun, H. Bai and G. Shi, ACS Nano, 2010, 4, 7358-7362.

73 A. Ghosh, K. V. Rao, S. J. George and C. N. R. Rao, Chem.-Eur. J., 2010, 16, 2700-2704.

74 J. Li, C. Liu and Y. Liu, J. Mater. Chem., 2012, 22, 8426-8430. 75 S. Li, S. Guo, H. Yang, G. Gou, R. Ren, J. Li, Z. Dong, J. Jin and J. Ma, J. Hazard. Mater., 2014, 270, 11-17.

76 J. Luo, N. Zhang, R. Liu and X. Liu, RSC Adv., 2014, 4, 6481664824.

77 Z. Zhang, H. Chen, C. Xing, M. Guo, F. Xu, X. Wang, H. J. Gruber, B. Zhang and J. Tang, Nano Res., 2011, 4, 599-611.

78 D. Briggs, Handbook of X-ray and Ultraviolet Photoelectron Spectroscopy, Heyden, London, 1977.

79 Y. Wang, H. Sauriat-Dorizon and H. Korri-Youssoufi, Sens. Actuators, B, 2017, 251, 40-48.

$80 \mathrm{Y}$. Hu, K. Wang, Q. Zhang, F. Li, T. Wu and L. Niu, Biomaterials, 2012, 33, 1097-1106. 